\title{
AUTO-APPLICATIONS HOLOMORPHES PROPRES DES DOMAINES POLYNOMIAUX RIGIDES DE $\mathrm{C}^{2}$
}

\author{
Abdelaziz Chaouech
}

\begin{abstract}
We show that proper holomorphic self maps of pseudoconvex rigid polynomial domains in $\mathbf{C}^{2}$ are automorphisms.
\end{abstract}

\section{Introduction}

Les seuls exemples connus d'applications holomorphes propres d'un domaine borné à bord régulier de $\mathbf{C}^{n}(n>1)$ sur lui-même sont des automorphismes. Ce phénomène constraste avec la situation unidimensionnelle et l'on présume son caractère général. Les domaines strictement pseudoconvexes vérifient cette conjecture: $\mathrm{H}$. Alexander $[\mathbf{1}]$ a traité le cas de la boule euclidienne et S. Pinchuk [13], celui des domaines arbitraires à bord de classe $\mathcal{C}^{2}$. De façon plus générale, K. Diederich et J. E. Fornaess ont mis en évidence le principe suivant: le lieu de branchement d'une application holomorphe propre d'un domaine pseudoconvexe borné sur un autre est vide dès que l'ensemble des points de faible pseudoconvexité de la frontière du domaine source est assez petit (cf. [10, Théorème 3]). Cependant, ce principe ne permet pas de traiter le cas des domaines faiblement pseudoconvexes dans sa généralité. A ce jour, seuls quelques cas ont été abordés. Ainsi, E. Bedford et S. Bell [2] ont considéré le cas des domaines pseudoconvexes bornés à bords analytiques réels et Y. Pan [12] celui des domaines de Reinhardt de type fini (voir aussi [9] où la preuve de Y. Pan est simplifiée et [8] où $\mathrm{F}$. Berteloot et S. Pinchuk classifient les applications holomorphes propres entre domaines de Reinhardt complets de $\mathbf{C}^{2}$ ). L'objet de cet article est d'étendre ce type de résultats à une classe de domaines faiblement pseudoconvexes non bornés. Notre principal résultat est le suivant: 
Théorème 1. Soit $P(z, \bar{z})$ un polynôme sous-harmonique sans terme harmonique. Soit $\Omega$ un domaine de $\mathbf{C}^{2}$ défini par $\Omega=:\left\{(w, z) \in \mathbf{C}^{2}\right.$ : $\operatorname{Re} w+P(z, \bar{z})<0\}$. Alors toute application holomorphe propre de $\Omega$ sur lui-même est un automorphisme.

Le principe de base de la démonstration est analogue à celui utilisé par Y. Pan. Nous exploitons simultanément la finitude du type de la frontière et l'existence d'un groupe à un paramètre d'automorphismes du domaine afin de préciser la structure du lieu de branchement de l'application. Cependant, comme les domaines que nous considérons sont non bornés et présentent moins de symétries que les domaines de Reinhardt, de nouvelles difficultés surgissent. En particulier, il est impossible d'établir directement que le lieu de branchement possède au plus un nombre fini de composantes connexes. Ainsi, après avoir montré que le lieu de branchement est contenu dans une réunion dénombrable de droites complexes (partie 1), nous sommes amenés à analyser certains biholomorphismes locaux induits par l'application (partie 2), puis certaines applications holomorphes propres entre domaines rigides distincts (partie 3). Nous pouvons ensuite montrer que - génériquement - l'application est conjuguée par des automorphismes de $\mathbf{C}^{2}$ à une application de la forme $(w, h(z))$ où $h$ est une fonction entière. Nous en déduisons que le nombre de composantes connexes du lieu de branchement est en fait majoré par le degré du polynôme $P$ (partie 4 ). Pour mener à bien cette dernière partie de la démonstration, nous établisons le résultat suivant:

Théorème 2. Soient $\Omega_{1}$ et $\Omega_{2}$ deux domaines de la forme $\Omega_{j}=$ $\left\{(w, z) \in \mathbf{C}^{2}: \operatorname{Re} w+P_{j}(z, \bar{z})<0\right\}$ où $P_{1}$ et $P_{2}$ sont des polynômes sous-harmoniques sans terme harmonique. Soit $f: \Omega_{1} \rightarrow \Omega_{2}$ une application holomorphe propre telle que:

1) $f=\left(f_{1}, f_{2}\right)$ se prolonge en un biholomorphisme local d'un voisinage de l'origine de $\mathbf{C}^{2}$ sur un autre.

2) $f_{2}(w, 0) \equiv 0$ et $f_{1}(0,0)=0$.

L'un des trois cas suivants est alors satisfait:

i) $f_{1}(w, z)=\Gamma w ;(\Gamma>0)$ et $f_{2}(w, z)=f_{2}(z)$.

ii) $f_{1}(w, z)=\Gamma w ;(\Gamma>0)$ et $P_{2}(z, \bar{z})=P_{2}(|z|,|z|)$.

iii) $f_{1}(w, z)=\frac{\Gamma w}{1+i \lambda w} ;\left(\Gamma>0, \lambda \in \mathbf{R}^{*}\right)$ et $P_{2}(z, \bar{z})=M|z|^{2 m}$ avec $M>0, m \in \mathbf{N}^{*}$.

Dans la suite de cet article, $\mathcal{P}$ désignera l'ensemble des polynômes sousharmoniques sans terme harmonique sur $\mathbf{C}$. 


\section{La structure du lieu de branchement}

L'objet de cette partie est d'établir la proposition suivante où l'on décrit le lieu de branchement des applications holomorphes propres du type de celles considérées dans cet article.

Proposition 1.1. Soient $\Omega_{1}$ et $\Omega_{2}$ deux domaines de $\mathbf{C}^{2}$ de la forme: $\Omega_{j}=\left\{(w, z) \in \mathbf{C}^{2}: \operatorname{Re} w+P_{j}(z, \bar{z})<0\right\}$ où $P_{1}$ et $P_{2}$ appartiennent à $\mathcal{P}$.

Pour toute application holomorphe propre de $\Omega_{1}$ sur $\Omega_{2}$, il existe une suite $\left(z_{n}\right)_{n}$ de nombres complexes telle que:

$$
V_{f}=\cup_{n \in N}\left\{\left(w, z_{n}\right): \operatorname{Re} w<-P_{1}\left(z_{n}, \bar{z}_{n}\right)\right\} .
$$

La démonstration de cette proposition repose essentiellement sur les deux lemmes énoncés ci-dessous. Le premier précise la structure locale de $\bar{V}_{f} \cap b \Omega_{1}$. Le second exhibe des fonctions p.s.h. négatives de $\Omega_{1}$ et $\Omega_{2}$ qui permettront de déduire la structure de $V_{f}$ de celle que $\bar{V}_{f} \cap b \Omega_{1}$.

Lemme 1.2. Soit $f: \Omega_{1} \rightarrow \Omega_{2}$ une application holomorphe propre satisfaisant les hypothèses de la Proposition 1.1 et soit $\left(w_{0}, z_{0}\right)$ un point de $b \Omega_{1}$.

Si l'on a

$$
\left(w_{0}, z_{0}\right) \in \bar{V}_{f} \cap b \Omega_{1} \text { et } \lim _{(w, z) \rightarrow\left(w_{0}, z_{0}\right)}\left[\left|f_{1}(w, z)\right|+\left|f_{2}(w, z)\right|\right]<+\infty
$$

alors l'ensemble $\left\{\left(w, z_{0}\right)\right.$ tel que $\left.\operatorname{Re} w<-P_{1}\left(z_{0}, \bar{z}_{0}\right)\right\}$ est contenu dans $V_{f}$.

Lemme 1.3. Soit $\Omega=:\left\{(w, z) \in \mathbf{C}^{2}: \operatorname{Re} w+P(z, \bar{z})<0\right\}$ où $P \in \mathcal{P}$. Alors il existe une fonction p.s.h. $\sigma: \Omega \rightarrow]-\infty, 0[$, telle que $\lim _{(|w|+|z|) \rightarrow+\infty} \sigma(w, z)=-\infty$. De plus, pour toute suite de nombres complexes $\left(z_{n}\right)_{n \leq 1}$, tout $a \notin\left\{z_{n} ; n>1\right\}$ et tout $\left(w_{a}, a\right) \in \Omega$, la fonction $\sigma$ peut être assujettie à satisfaire les conditions suivantes:

1) $\sigma\left(w_{a}, a\right) \neq-\infty$.

2) $\forall n \geq 1, \forall\left(w_{n}, z_{n}\right) \in b \Omega, \lim _{(w, z) \rightarrow\left(w_{n}, z_{n}\right)} \sigma(w, z)=-\infty$.

(Dans ce cas la fonction $\sigma$ prend ses valeurs dans $[-\infty, 0[$.

Preuve de la Proposition 1.1: Supposons $V_{f}$ non vide. Soit

$$
A=\left\{\left(w_{0}, z_{0}\right) \in \bar{V}_{f} \cap b \Omega_{1}: \lim _{(w, z) \rightarrow\left(w_{0}, z_{0}\right)}\left[\left|f_{1}(w, z)\right|+\left|f_{2}(w, z)\right|\right]<+\infty\right\}
$$


et $A_{2}$ la projection de $A$ sur le plan de la variable $z$. Le Lemme 1.2 montre que $A_{2}$ est localement fini. En effet, si cela n'était pas, l'ensemble analytique $V_{f}$ contiendrait une famille de demi-plans de la forme $\left\{\left(w, z_{p}\right)\right.$ : $\left.\operatorname{Re} w<-P_{1}\left(z_{p}, \bar{z}_{p}\right)\right\},\left(z_{p}\right)$ étant convergente modulo extraction. Ces demi-plans s'accumuleraient nécessairement sur un demi-plan de la même forme. On en déduirait facilement que $V_{f}=\Omega_{1}$ ce qui est impossible. Ainsi $A_{2}$ est dénombrable et nous noterons $\left(z_{n}\right)_{n \geq 1}$ la suite ordonnée de ses éléments.

Soit $\mathcal{C}$ une composante connexe de $V_{f}$. Nous achèverons la démonstration en montrant que $\mathcal{C}$ coïncide avec un demi-plan de la forme $\left\{\left(w, z_{n}\right)\right.$ : $\left.\operatorname{Re} w<-P_{1}\left(z_{n}, \bar{z}_{n}\right)\right\}$ où $z_{n} \in A_{2}$. Pour cela, procédons par l'absurde et supposons qu'il existe $\left(w_{a}, a\right) \in \mathcal{C}$ tel que $a \notin\left\{z_{n}, n \geq 1\right\}$.

Soit $\sigma_{1}$ une fonction p.s.h. et négative sur $\Omega_{1}$ associée par le Lemme 1.3 à la suite $\left(z_{n}\right)_{n \geq 1}$ et au point $\left(w_{a}, a\right)$. D'après le même lemme, il existe également une fonction $\sigma_{2}$, p.s.h. négative sur $\Omega_{2}$ et telle que $\lim _{(|w|+|z|) \rightarrow+\infty} \sigma_{2}(w, z)=-\infty$. Considérons alors la fonction $\tilde{\sigma}$ définie par $\tilde{\sigma}=: \exp \left(\sigma_{1}+\sigma_{2} \circ f\right)$. Cette fonction est p.s.h. sur $\Omega_{1}$ et prend ses valeurs dans $[0,1]$. De plus, elle satisfait les propriétés suivantes:

1) $0 \leq \lim _{\substack{(|w|+|z|) \rightarrow+\infty \\(w, z) \in \mathcal{C}}} \tilde{\sigma}(w, z) \leq \lim _{(|w|+|z|) \rightarrow+\infty} e^{\sigma_{1}(w, z)}=0$.

2) $\operatorname{Si}\left(w_{n}, z_{n}\right) \in \overline{\mathcal{C}} \cap b \Omega_{1}$ et $z_{n} \in A_{2}$ alors on a

$$
0 \leq \lim _{\substack{(w, z) \rightarrow\left(w_{n}, z_{n}\right) \\(w, z) \in \mathcal{C}}} \tilde{\sigma}(w, z) \leq \lim _{(w, z) \rightarrow+\left(w_{n}, z_{n}\right)} e^{\sigma_{1}(w, z)}=0 .
$$

3) $\mathrm{Si}\left(w_{0}, z_{0}\right) \in \overline{\mathcal{C}} \cap b \Omega_{1}$ et $z_{0} \notin A_{2}$ alors par définition de $A$, on a:

$$
\begin{aligned}
& (w, z) \rightarrow\left(w_{0}, z_{0}\right) \\
& 0 \leq \lim _{\substack{(w, z) \rightarrow\left(w_{0}, z_{0}\right) \\
(w, z) \in \mathcal{C}}}\left[\left|f_{1}(w, z)\right|+f_{2}(w, z)\right]=+\infty \text { et donc } \\
& \sigma(w, z) \leq \lim _{(w, z) \rightarrow\left(w_{0}, z_{0}\right)} e^{\sigma_{2} \circ f(w, z)}=0 .
\end{aligned}
$$

Il résulte des conditions 1$), 2$ ) et 3 ) et du principe du maximum que la restriction de $\tilde{\sigma}$ à $\mathcal{C}$ est identiquement nulle. Ceci est absurde puisque, par construction, $\tilde{\sigma}\left(w_{a}, a\right)>0$. La preuve de la proposition est donc achevée.

Preuve du Lemme 1.3: Notons $2 m$ le degré du polynôme $P$ et posons

$$
Q_{z_{n}}(z, \bar{z})=: P\left(z+z_{n}, \bar{z}+\bar{z}_{n}\right)-2 \operatorname{Re} \sum_{j=1}^{2 m} \frac{1}{j !} \frac{\partial^{j} P}{\partial z^{j}}\left(z_{n}, \bar{z}_{n}\right) z^{j}-P\left(z_{n}, \bar{z}_{n}\right) .
$$


L'automorphisme de $\mathbf{C}^{2}$ défini par $\Phi_{z_{n}}(w, z)=\left(w^{\prime}, z^{\prime}\right)$ où $w^{\prime}=$ $w-2 \sum_{j=1}^{2 m} \frac{\partial^{j} P}{\partial z^{j}}\left(z_{n}, \bar{z}_{n}\right) z^{j}-P\left(z_{n}, z_{n}\right)$ et $z^{\prime}=z+z_{n}$ réalise un biholomorphisme du domaine $\Omega_{z_{n}}=:\left\{(w, z): \operatorname{Re} w+Q_{z_{n}}(z, \bar{z})<0\right\}$ sur $\Omega$.

Le polynôme $Q_{z_{n}}$ étant sous-harmonique et sans terme harmonique, sa partie homogène de plus haut degré l'est également, nous la noterons $H_{z_{n}}$.

Pour $\varepsilon>0$ fixé, on vérifie sans peine qu'il existe $a>0$ tel que $H_{z_{n}}(z, \bar{z})-Q_{z_{n}}(z, \bar{z})<a+\varepsilon|z|^{2 m}$ pour tout $z \in \mathbf{C}$. Ainsi $\bar{\Omega}_{z_{n}}$ est contenu dans un domaine $D_{z_{n}}$ défini par:

$$
D_{z_{n}}=:\left\{(w, z): \operatorname{Re}(w-a)+H_{z_{n}}(z ; \bar{z})-\varepsilon|z|^{2 m}<0\right\} .
$$

D'après E. Bedford et J. E. Fornaess ([3, Main Theorem $]$ ), il existe une fonction $g_{z_{n}}$ holomorphe sur $D_{z_{n}}$ et continue sur $\bar{D}_{z_{n}}$ satisfaisant les propriétés suivantes: pour $(w, z) \in \bar{D}_{z_{n}}$ et $N_{n} \in \mathbf{N}$ assez grand on a

i) $B_{n}\left(|w-a|+|z|^{2 m}\right) \leq\left|g_{z_{n}}(w, z)\right|^{N_{n}} \leq A_{n}\left(|w-a|+|z|^{2 m}\right)$ où $A_{n}$ et $B_{n}$ sont des constantes strictement positives.

ii) $\arg g_{z_{n}}(w, z) \in\left[-\frac{\pi}{4}, \frac{\pi}{4}\right]$.

Soit $g$ la fonction ainsi obtenue lorsque $z_{n}=0$.

En posant $\sigma=\log \left|\frac{1}{K}\left[1-\left(\frac{\alpha g-1}{\alpha g+1}\right)\right]\right|$ où $\alpha>0$ est assez petit et $K>0$ est assez grand, on obtient une fonction p.s.h. dans $\Omega$ prenant ses valeurs dans $]-\infty, 0$ [ et telle que $\lim _{(|w|+|z|) \rightarrow+\infty} \sigma(w, z)=-\infty$.

Nous terminons en modifiant la fonction $\sigma$ de façon à obtenir une fonction p.s.h. négative sur $\Omega$ prenant la valeur $-\infty$ sur les droites complexes $\left\{z=z_{n}\right\}$.

Posons, à cet effet, $\tilde{h}_{z_{n}}(w, z)=\frac{B_{n} z^{2 m}}{g_{z_{n}}^{N}(w, z)}$, on définit ainsi une fonction holomorphe de module strictement inférieur à 1 sur $D_{z_{n}}$. La fonction $h_{z_{n}}=: \tilde{h}_{z_{n}} \circ \Phi_{z_{n}}^{-1}$ est alors holomorphe au voisinage de $\bar{\Omega}$ et satisfait les propriétés suivantes:

i) $\forall(w, z) \in \bar{\Omega},\left|h_{z_{n}}(w, z)\right|<1$.

ii) $\forall(w, z) \in \bar{\Omega}$, on a $h_{z_{n}}(w, z)=0$ si et seulement si $z=z_{n}$.

Choisissons une suite $\lambda_{n}>0$ telle que $\sum_{n=1}^{+\infty} \lambda_{n} \log \left|h_{z_{n}}\left(w_{a}, a\right)\right|>-\infty$; alors $\sum_{n=1}^{+\infty} \lambda_{n} \log \left|h_{z_{n}}(w, z)\right|$ définit une fonction p.s.h. négative sur $\Omega$ et la fonction

$$
\sigma=\log \left|\frac{1}{K}\left[1-\left(\frac{\alpha g-1}{\alpha g+1}\right)\right]\right|+\sum_{n=1}^{+\infty} \lambda_{n} \log \left|h_{z_{n}}\right|
$$

remplit les conditions requises. 
Preuve du Lemme 1.2: Soit $\left(\left(w_{n}, z_{n}\right)\right)_{n \geq 1}$ une suite de points de $\Omega_{1}$ qui converge vers un point $\left(w_{0}, z_{0}\right)$ de $b \Omega_{1}$. Si $\underline{\lim }\left[\left|f_{1}\left(w_{n}, z_{n}\right)\right|+\right.$ $\left.\left|f_{2}\left(w_{n}, z_{n}\right)\right|\right]<+\infty$ alors, l'application $f$ étant propre, la suite $\left(f\left(w_{n}, z_{n}\right)\right)_{n \geq 1}$ converge vers un point $\left(w_{0}^{\prime}, z_{0}^{\prime}\right)$ de $b \Omega_{2}$ après une éventuelle extraction. Le bord de $\Omega_{2}$ étant de type fini, un résultat de F. Berteloot [6] montre que l'application $f$ se prolonge continûment à $\bar{\Omega}_{1}$ sur un voisinage de $\left(w_{0}, z_{0}\right)$. Nous aurons également besoin de la différentiabilité du prolongement de $f$, celle-ci découle des résultats de [5].

Pour tout $p \in b \Omega_{j},(j=1$ ou 2$)$, on note $\tau(p)$ l'ordre d'annulation du déterminant de Levi de $b \Omega_{j}$ en $p$. Plus précisément, pour toute fonction $\rho$, définissante locale de $b \Omega_{j}$ en $p$, on pose $\Lambda_{p}=-\operatorname{det}\left[\begin{array}{cc}0 & \rho_{\bar{z}_{k}} \\ \rho_{z_{i}} & \rho_{z_{i}} \bar{z}_{k}\end{array}\right]$ et $\tau(p)$ est le plus petit entier $m$ pour lequel existe un opérateur $T$, tangentiel au bord d'ordre $m$, tel que $T \Lambda_{\rho}(p) \neq 0$.

Ainsi, $\tau$ satisfait les propriétés suivantes (voir [12, page 291]):

1) $\tau$ est indépendant de la fonction définissante choisie.

2) $\tau$ est semi-continue supérieurement (s.c.s.).

3) $\tau$ est invariant par biholomorphisme.

4) $\forall p \in b \Omega$ on a $\tau(p) \geq \tau(f(p))$ et, en outre, $\tau(p)=\tau(f(p))$ si et seulement si $p \notin \bar{V}_{f}$.

On remarquera que lorsque $\Omega_{j}$ est un domaine défini par $\Omega_{j}=\{(w, z) \in$ $\mathbf{C}^{2}$ tel que $\left.\operatorname{Re} w+P_{j}(z \bar{z})<0\right\}$ où $P_{j} \in \mathcal{P}$ alors, pour tout $(w, z) \in b \Omega_{j}$, $\tau(w, z)$ n'est autre que l'ordre d'annulation du Laplacien de $P_{j}$ au point $z$.

Nous sommes maintenant en mesure de terminer la preuve du lemme. Il suffit d'établir que $J_{f}\left(w_{0}+i t, z_{0}\right)$ est identiquement nul au voisinage de $t=0$. Si cela n'était pas, on trouverait une suite $\left(t_{n}\right)_{n \in N}$ telle que $\lim _{n} t_{n}=0$ et $J_{f}\left(w_{0}+i t_{n}, z_{0}\right) \neq 0$.

Pour tout $n \geq 1$, on aurait alors $\tau\left[f\left(w_{0}+i t_{n}, z_{0}\right)\right]=\tau\left[\left(w_{0}+i t_{n}, z_{0}\right)\right]=$ $\tau\left(w_{0}, z_{0}\right)$ et $\tau$ étant s.c.s., $\tau\left(w_{0}, z_{0}\right) \leq \tau\left(f\left(w_{0}, z_{0}\right)\right)$. Comme, par ailleurs, on a $\tau\left(f\left(w_{0}, z_{0}\right)\right) \leq \tau\left(w_{0}, z_{0}\right)$, il en résulterait que $\tau\left(f\left(w_{0}, z_{0}\right)\right)=\tau\left(w_{0}, z_{0}\right)$ et donc que $J_{f}\left(w_{0}, z_{0}\right) \neq 0$, ce qui est contraire aux hypothèses.

\section{2. Étude de certains biholomorphismes locaux}

Nous étudions ici les biholomorphismes locaux échangeant deux germes d'hypersurfaces analytiques réelles, rigides et de type finis. Nous supposons en outre que ces biholomorphismes préservent une droite complexe transverse aux hypersurfaces. La proposition suivante résume les résultats de cette partie. 
Proposition 2.1. Soit $f=\left(f_{1}, f_{2}\right)$ un biholomorphisme local d'un voisinage $V$ de l'origine dans $\mathbf{C}^{2}$ sur un autre et tel que $f_{1}(0,0)=0$, $f_{2}(w, 0) \equiv 0$.

Soient deux hypersurfaces $H_{1}$ et $H_{2}$ définies par:

$$
\begin{aligned}
& H_{1}=\left\{\rho_{1}(w, z)=: \operatorname{Re} w+\varphi(z, \bar{z})=0\right\} . \\
& H_{2}=\left\{\rho_{2}(w, z)=: \operatorname{Re} w+\Psi(z, \bar{z})=0\right\} .
\end{aligned}
$$

où $\varphi$ et $\Psi$ sont des fonctions analytiques réelles sous-harmoniques défnies au voisinage de l'origine, telles que: $\frac{\partial^{k} \varphi}{\partial z^{k}}(0,0)=0, \frac{\partial^{k} \Psi}{\partial z^{k}}(0,0)=0$ pour tout $k \geq 0$ et $\frac{\partial^{2} \Psi}{\partial z \partial \bar{z}}, \frac{\partial^{2} \varphi}{\partial z \partial \bar{z}}$ s'annulent $\grave{a}$ un ordre fini en 0 . On suppose que $f\left(H_{1} \cap V\right) \subset H_{2}$. Alors:

a) $f_{1}$ ne dépend que de $w$.

b) Si, de plus, $f_{1}(w)=\Gamma w(\Gamma>0)$ alors:

$f_{*}\left(i \frac{\partial}{\partial w}\right)=i \Gamma \frac{\partial}{\partial w}+B(z) \frac{\partial}{\partial z}$ où $B$ est une fonction holomorphe en $z$ telle que $B(0)=0$ et $B^{\prime}(0)=i \beta, \beta \in \mathbf{R}$. La fonction $B$ est identiquement nulle, si et seulement si $B^{\prime}(0)=0$.

Preuve de a): Notons $u$ et $v$ les parties réelles et imaginaires de la variable $w$. Soit $A(v, z)=:(-\varphi(z)+i v, z)$ un paramétrage de $H_{1}$. Le champ de vecteurs $L$ défini par $L=:-\frac{\partial \varphi}{\partial z} \frac{\partial}{\partial w}+\frac{1}{2} \frac{\partial}{\partial z}$ est tangent à $H_{1}$ et donc $L\left(\rho_{2} \circ f\right) \equiv 0$ sur $H_{1} \cap V$. En posant $g(w, z)=\frac{\partial}{\partial w}\left(\rho_{2} \circ f\right)=$ $\frac{1}{2} \frac{\partial f_{1}}{\partial w}+\frac{\partial}{\partial w}\left(\Psi \circ f_{2}\right)$, on obtient:

(1) $\left(-\frac{\partial \varphi}{\partial z}\right) \cdot(g \circ A)+\frac{1}{2}\left[\frac{1}{2} \frac{\partial f_{1}}{\partial z} \circ A+\frac{\partial \Psi}{\partial z} \circ\left(f_{2} \circ A\right) \cdot \frac{\partial f_{2}}{\partial z} \circ A\right] \equiv 0$

au voisinage de $v=0, z=0$.

La démonstration consiste à dériver (1) par rapport à $z$ à un ordre arbitraire.

Començons par quelques préliminaires. En observant que, d'après la définition de $A$, l'on a

$$
\frac{\partial}{\partial z}\left(\bar{f}_{2} \circ A\right)=-\left(\frac{\partial \bar{f}_{2}}{\partial \bar{w}} \circ A\right) \cdot \frac{\partial \varphi}{\partial z}
$$

et

$$
\frac{\partial}{\partial z}\left(f_{2} \circ A\right)=-\left(\frac{\partial f_{2}}{\partial w} \circ A\right) \cdot \frac{\partial \varphi}{\partial z}+\left(\frac{\partial f_{2}}{\partial z} \circ A\right)
$$


on obtient

(2) $\frac{\partial}{\partial z}\left[\frac{\partial^{j} \Psi}{\partial z^{j}} \circ\left(f_{2} \circ A\right)\right]=\left[\frac{\partial^{j+1} \Psi}{\partial z^{j+1}} \circ\left(f_{2} \circ A\right)\right]\left[\frac{\partial f_{2}}{\partial z} \circ A\right]$
$-\frac{\partial \varphi}{\partial z}\left[\left(\frac{\partial f_{2}}{\partial w} \circ A\right) \cdot \frac{\partial^{j+1} \Psi}{\partial z^{j+1}} \circ\left(f_{2} \circ A\right)+\left(\frac{\partial \bar{f}_{2}}{\partial \bar{w}} \circ A\right) \frac{\partial^{j+1} \Psi}{\partial \bar{z} \partial z^{j}} \circ\left(f_{2} \circ A\right)\right]$.

On montre maintenant par récurrence que

$$
\begin{aligned}
& \text { (3) } \frac{\partial^{k}}{\partial z^{k}}\left[\frac{\partial \Psi}{\partial z} \circ\left(f_{2} \circ A\right)\right]=\left[\frac{\partial^{k+1} \Psi}{\partial z^{k+1}} \circ\left(f_{2} \circ A\right)\right]\left[\frac{\partial f_{2}}{\partial z} \circ A\right]^{k} \\
& +\sum_{j=1}^{k}\left[g_{j}\left(\frac{\partial^{j} \Psi}{\partial z^{j}} \circ\left(f_{2} \circ A\right)\right)+h_{j} \frac{\partial^{j} \varphi}{\partial z^{j}}\right] \text {. }
\end{aligned}
$$

Les fonctions $g_{j}$ et $h_{j}$ sont analytiques réelles au voisinage de $(0,0)$ mais nous ne cherchons pas à les expliciter.

Pour $k=1$, il s'agit de la formule (2) avec $j=1$ et $g_{1} \equiv 0$. En utilisant (2), on voit immédiatement que

$$
\begin{aligned}
\frac{\partial}{\partial z}\left[\sum_{j=1}^{k} g_{j}\left(\frac{\partial^{j} \Psi}{\partial z^{j}} \circ\left(f_{2} \circ A\right)\right)\right. & \left.+h_{j} \frac{\partial^{j} \varphi}{\partial z^{j}}\right] \\
& =\sum_{j=1}^{k+1} \tilde{g}_{j}\left(\frac{\partial^{j} \Psi}{\partial z^{j}} \circ\left(f_{2} \circ A\right)\right)+\tilde{h}_{j} \frac{\partial^{j} \varphi}{\partial z^{j}} .
\end{aligned}
$$

Il reste donc à noter que:

$$
\begin{aligned}
\frac{\partial}{\partial z}\left\{\left[\frac{\partial^{k+1} \Psi}{\partial z^{k+1}} \circ\left(f_{2} \circ A\right)\right]\left[\frac{\partial f_{2}}{\partial z} \circ A\right]^{k}\right\} & {\left[\frac{\partial}{\partial z}\left(\frac{\partial^{k+1} \Psi}{\partial z^{k+1}} \circ\left(f_{2} \circ A\right)\right)\right]\left[\frac{\partial f_{2}}{\partial z} \circ A\right]^{k} } \\
+ & {\left[\frac{\partial^{k+1} \Psi}{\partial z^{k+1}} \circ\left(f_{2} \circ A\right)\right]\left[\frac{\partial}{\partial z}\left(\frac{\partial f_{2}}{\partial z} \circ A\right)^{k}\right] . }
\end{aligned}
$$

D'autre part, une récurrence immédiate donne:

$$
\frac{\partial^{k}}{\partial z^{k}}\left[\frac{\partial f_{1}}{\partial z} \circ A\right]=\left(\frac{\partial^{k+1} f_{1}}{\partial z^{k+1}} \circ A\right)+\sum_{j=1}^{k}\left(\frac{\partial^{j} \varphi}{\partial z^{j}}\right) l_{j} .
$$


Là encore, les $l_{j}$ sont des fonctions que nous n'explicitons pas. En utilisant l'hypothèse $f_{2}(w, 0) \equiv 0$, on tire de $(3)$ et $(4)$ :

$$
\begin{aligned}
& \frac{\partial^{k}}{\partial z^{k}}\left[\frac{\partial \Psi}{\partial z} \circ\left(f_{2} \circ A\right)\right](i v, 0) \equiv 0, \text { pour } k \geq 0 ; \\
& \frac{\partial^{k}}{\partial z^{k}}\left[\frac{\partial f_{1}}{\partial z} \circ A\right](i v, 0)=\frac{\partial^{k+1} f_{1}}{\partial z^{k+1}}(i v, 0), \text { pour } k \geq 0
\end{aligned}
$$

En appliquant $\frac{\partial^{k}}{\partial z^{k}}$ à l'équation (1) et en tenant compte de (5), (6), on trouve alors $\frac{\partial^{k+1} f_{1}}{\partial z^{k+1}}(i v, 0) \equiv 0$ pour $k \geq 0$, ce qui établit a).

Passons maintenant à la preuve de b).

Preuve de b): Nous utiliserons des champs de vecteurs holomorphes tangents aux hypersurfaces $H_{j}$, ce qui désignera ici des champs de la forme $a(w, z) \frac{\partial}{\partial w}+b(w, z) \frac{\partial}{\partial z}$ où les fonctions $a, b$ sont holomorphes et dont les parties réelles sont des champs tangents au sens usuel. Rappelons que cette classe de champs de vecteurs est stable par image directe par un biholomorphisme ainsi que par crochet de Lie. La proposition suivante, extraite de [4], sera utile à la démonstration. Pour la commodité de lecteur, nous en donnerons la preuve à la fin de cette partie.

Proposition 2.2. Soit $\vec{X}=h(w, z) \frac{\partial}{\partial z}$ un champ de vecteur holomorphe tangent à $\mathrm{H}_{2}$ défini au voisinage de l'origine et non identiquement nul. Alors la partie homogène de plus bas degré de $h$ en $z$ est égale à $i \beta z$ $\left(\beta \in \mathbf{R}^{*}\right)$ et celle de $\Psi$ à $M|z|^{2 m}$, avec $M>0$ et $m \in \mathbf{N}^{*}$.

Notons $f(w, z)=:\left(\Gamma w, f_{2}(w, z)\right)$ et considérons le champ de vecteur holomorphe tangent à $H_{2}$ c'est-à-dire $f_{*}\left(i \frac{\partial}{\partial w}\right)=: A(w, z) \frac{\partial}{\partial w}+B(w, z) \frac{\partial}{\partial z}$.

On a $\left[f_{*}\left(i \frac{\partial}{\partial w}\right)\right]_{f(w, z)}=i \frac{\partial f_{1}}{\partial w} \frac{\partial}{\partial w}+i \frac{\partial f_{2}}{\partial w} \frac{\partial}{\partial z}$ d'où

$$
B\left(\Gamma w, f_{2}(w, z)\right)=i \frac{\partial f_{2}}{\partial w}(w, z) \text { et } A \equiv i \Gamma .
$$

Puisque $\Gamma i \frac{\partial}{\partial w}$ est tangent à $H_{2}, B(w, z) \frac{\partial}{\partial z}$ l'est également.

Supposons $B$ non identiquement nul. Alors en appliquant la Proposition 2.2 aux champs $B(w, z) \frac{\partial}{\partial z}$ et $\left[i \frac{\partial}{\partial w}, B(w, z) \frac{\partial}{\partial z}\right]=i \frac{\partial B}{\partial w} \frac{\partial}{\partial z}$, on voit que le développement de $B$ est de la forme suivante:

$$
\begin{aligned}
B(w, z)=\left(i \beta z+\sum_{k \geq 2} b_{k}^{0} z^{k}\right)+\alpha w & \left(z+\sum_{k \geq 2} b_{k}^{1} z^{k}\right) \\
& +\sum_{q \geq 2} c_{q} w^{q}\left(z+\sum_{k \geq 2} b_{k}^{q} z^{k}\right),
\end{aligned}
$$


où $\beta$ et $\alpha$ sont des réels qui ne sont respectivement nuls que si $B \equiv 0$ ou $\frac{\partial B}{\partial w} \equiv 0$.

Ecrivons le développement de $f_{2}(w, z)$ sous la forme suivante

$\begin{aligned} \text { (9) } f_{2}(w, z)= & \left(a z+\sum_{k \geq 2} a_{k}^{0} z^{k}\right)+w\left(b z+\sum_{k \geq 2} a_{k}^{1} z^{k}\right) \\ & +w^{2}\left(c z+\sum_{k \geq 2} a_{k}^{2} z^{k}\right)+\sum_{q \geq 3} w^{q}\left(d_{q} z+\sum_{k \geq 2} a_{k}^{q} z^{k}\right) .\end{aligned}$

En identifiant les termes en $z$ puis en $w z$ dans chacun des deux membres de (7), on obtient: $i b=i \beta a$ et $2 i c=i \beta b+\alpha \Gamma a$ puis donc $b=\beta a$ et $c=\gamma a$, où $\gamma=\frac{1}{2}\left(\beta^{2}-i \alpha \Gamma\right)$.

Traduisons maintenant l'inclusion $f\left(H_{1}\right) \subset H_{2}$ :

$$
\Psi\left[f_{2}(-\varphi+i v, z)\right] \equiv \Gamma \varphi(z, \bar{z})
$$

Les termes de degré 1 en $z$ dans $f_{2}(-\varphi+i v, z)$ proviennent de:

$$
a z+b(-\varphi+i v) z+c(-\varphi+i v)^{2} z+\sum_{q \geq 3} d_{q}(-\varphi+i v)^{q} z .
$$

La partie de degré 1 en $z$ dans $f_{2}(-\varphi+i v, z)$ est donc égale à: $a z(1+$ $\left.\beta i v-\gamma v^{2}+o\left(v^{2}\right)\right)$.

D'après la Proposition 2.2, la partie homogène de plus bas degré dans $\Psi$ est égale à $B|z|^{2 m}(B>0)$. Par symétrie, celle de $\varphi$ est égale à $A|z|^{2 m}$ $(A>0)$. L'identité des termes de degré $2 m$ en $z$ dans (10) donne alors:

$$
B|a|^{2 m}|z|^{2 m}\left|1+\beta i v-\gamma v^{2}+o\left(v^{2}\right)\right|^{2 m} \equiv \Gamma A|z|^{2 m},
$$

d'où:

$$
\left|1+\beta i v-\gamma v^{2}+o\left(v^{2}\right)\right|^{2} \equiv 1
$$

et donc: $\left(\beta^{2}-2 \gamma\right) v^{2}+o\left(v^{2}\right)=i \alpha \Gamma v^{2}+o\left(v^{2}\right) \equiv 0$.

Il s'ensuit que $\alpha=0$ et donc $\frac{\partial B}{\partial w} \equiv 0$. Ceci achève la preuve de la Proposition 2.1.

Nous terminons cette partie en donnant la démonstration de la Proposition 2.2. Cette dernière résulte du lemme technique suivant: 
Lemme 2.3. Soit $Q(z, \bar{z})$ un polynôme à valeurs réelles homogène de degré $2 m$, sans terme harmonique, et soit $(\lambda, k) \in \mathbf{R} \times \mathbf{N}$.

1) $S i \operatorname{Im}\left(z^{k} \frac{\partial Q}{\partial z}\right)=\lambda \operatorname{Re}\left(z^{k} \frac{\partial Q}{\partial z}\right)$; alors:

i) Si $k=1$, on $a=M|z|^{2 m}(M>0)$ et $\lambda=0$.

ii) $S i k \neq 1$ on $a \equiv 0$.

2) Si $\operatorname{Re}\left(z^{k} \frac{\partial Q}{\partial z}\right)=0$, alors $Q=0$.

Començons par la preuve du Lemme 2.3.

Preuve du Lemme 2.3: Notons:

$$
Q=\sum_{\substack{p+q=2 m \\ p, q \geq 1}} A_{p q} z^{p \bar{z}^{p}} \text { et } Q_{1}=\frac{\partial Q}{\partial z}
$$

On a alors, en posant $l=k-1$,

$$
z^{k} Q_{1}=\sum_{\substack{p+q=2 m \\ p, q \geq 1}} p A_{p q} z^{p+l} \bar{z}^{q}
$$

et

$$
\bar{z}^{k} \bar{Q}_{1}=\sum_{\substack{p+q=2 m \\ p, q \geq 1}} q A_{p q} z^{p \bar{z}^{q+l}}
$$

On a, par hypothèse:

$$
\frac{1}{2 i}\left(z^{k} Q_{1}-\bar{z}^{k} \bar{Q}_{1}\right)=\frac{\lambda}{2}\left(z^{k} Q_{1}+\bar{z}^{k} \bar{Q}_{1}\right)
$$

ou encore:

$$
\sum_{\substack{p+q=2 m \\ p, q \geq 1}} p(1-\lambda i) A_{p q} z^{p+l} \bar{z}^{q}=\sum_{\substack{p+q=2 m \\ p, q \geq 1}} q(1+\lambda i) A_{p q} z^{p} \bar{z}^{q+l} .
$$

Lorsque $k \neq 1$ (i.e. $l \neq 0$ ), on voit directement sur (11) que $Q \equiv 0$. En effet, si $p_{0}=\min \left\{p \in[1,2 m-1]\right.$ tels que $\left.A_{p, q} \neq 0, q+p=2 m\right\}$ alors les termes de plus petit degré en $z$ de chacun de deux membres de $(11)$ sont respectivement égaux à $p_{0}(1-\lambda i) A_{p_{0} q_{0}} z^{p_{0}+l} \bar{z}^{q_{0}}$ et $q_{0}(1+$

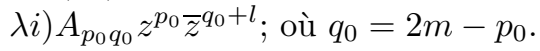

Lorsque $k=1$ (i.e. $l=0$ ) (11) devient:

$$
\sum_{\substack{p+q=2 \\ p, q \geq 1}}[p(1-\lambda i)-q(1+\lambda i)] A_{p q} z^{p} \bar{z}^{q} \equiv 0,
$$


d'où $A_{p q}=0$ pour $p \neq q$ et, si $Q \not \equiv 0, \lambda=0$.

Ceci établit la première assertion. La seconde s'obtient de façon analogue.

Preuve de la Proposition 2.2: Soit $Q$ la partie homogène de plus bas degré dans le développement de $\Psi$ au voisinage de l'origine. En vertu des hypothèses sur $\Psi, Q(z, \bar{z})$ est un polynême de degré $2 m$ sous-harmonique et sans terme harmonique.

Donnons à $w($ et $\bar{w})$ le poids $2 m$ et à $z($ et $\bar{z})$ le poids 1 . Ainsi le poids d'un monôme $\omega^{k_{1}} \bar{w}^{k_{2}} z^{q_{1}} \bar{z}^{q_{2}}$ est égal à $\left(k_{1}+k_{2}\right) 2 m+\left(q_{1}+q_{2}\right)$.

Soit $B(w, z)$ la partie homogène de plus bas poids dans le développement de $h$ au voisinage de $(0,0)$. Le champ $h(w, z) \frac{\partial}{\partial z}$ est holomorphe tangent à $\mathrm{H}_{2}$ c'est-à-dire:

$$
\operatorname{Re}\left[h(-\Psi(z, \bar{z})+i v, z) \frac{\partial \Psi}{\partial z}(z, \bar{z})\right] \equiv 0 .
$$

En notant $Q_{1}=\frac{\partial Q}{\partial z}$, et en collectant les termes de plus bas degré en $z$ dans (13), on obtient:

$$
\operatorname{Re}\left[B(-Q+i v, z) Q_{1}\right] \equiv 0
$$

Supposons que $B$ soit de poids $q$ où $q \in\{0, \ldots, 2 m-1\}$. On a donc $B(w, z)=\gamma z^{q}$ où $\gamma=\alpha+i \beta \neq 0$ et (14) donne: $\alpha \operatorname{Re}\left(z^{q} Q_{1}\right)=$ $\beta \operatorname{Im}\left(z^{q} Q_{1}\right)$. Le Lemme 2.3 montre alors que $q=1, \alpha=0$ et $Q=$ $M|z|^{2 m}$.

Si maintenant $B$ est de degré $q \geq 2 m$, on a $B(w, z)=b_{0} z^{q}+b_{1} w z^{q-2 m}+$ $\cdots+b_{s} w^{s} z^{q-2 m s}$ l'équation (14) devient:

(15) $\operatorname{Re}\left[Q_{1}\left(b_{0} z^{q}+b_{1} z^{q-2 m}(-Q+i v)+\cdots+b_{s} z^{q-2 m s}(-Q+i v)^{s}\right)\right] \equiv 0$.

Supposons d'abord $s>0$, en derivant $s$ fois l'équation (15) par rapport à $v$, on obtient:

$$
\operatorname{Re}\left[b_{s} ! i^{s} z^{q-2 m s} Q_{1}\right] \equiv 0
$$

et le Lemme 2.3 montre que:

$$
Q=M|z|^{2 m} ; q=2 m s+1 \text { et } b_{s} s ! i^{s}=i \lambda, \text { où } \lambda \in \mathbf{R}^{*} .
$$

Dérivons maintenant $(s-1)$ fois l'équation (15) par rapport à $v$, on obtient:

$\operatorname{Re}\left\{\left[b_{s-1} i^{s-1}(s-1) ! z^{q-2 m(s-1)}+b_{s} i^{s-1} s !(-Q+i v) z^{q-2 m s}\right] Q_{1}\right\} \equiv 0$

d'où, en tenant compte de (16):

$\operatorname{Re}\left[b_{s-1} i^{s-1}(s-1) ! z^{2 m}\left(m M|z|^{2 m}\right)-\lambda m M^{2}|z|^{4 m}+\lambda i v\left(m M|z|^{2 m}\right)\right] \equiv 0$.

On a alors $\lambda m \cdot M^{2}=0$, ce qui est absurde puisque $b_{s} \neq 0$. Lorsque $s=0$, la contradiction découle immédiatement du Lemme 2.3. Ceci termine la preuve de la Proposition 2.2. 


\section{Applications holomorphes propres entre domaines polynomiaux rigides et fixant une droite complexe}

Dans cette partie, nous étudions la forme des applications holomorphes propres entre deux domaines polynomiaux rigides de $\mathbf{C}^{2}$ qui fixent une droite de la forme $\{z=$ cte $\}$ non contenue dans le lieu de branchement. Nous établisons la proposition suivante.

Proposition 3.1. Soient $\Omega_{1}$ et $\Omega_{2}$ deux domaines de la forme: $\Omega_{j}=$ $\left\{(w, z) \in \mathbf{C}^{2}: \operatorname{Re} w+P_{j}(z, \bar{z})<0\right\}$ où $P_{1}$ et $P_{2}$ appartiennent à $\mathcal{P}$.

Soit $f: \Omega_{1} \rightarrow \Omega_{2}$ une application holomorphe propre telle que:

1) $f=\left(f_{1}, f_{2}\right)$ se prolonge en un biholomorphisme local d'un voisinage $U_{1}$ de l'origine de $\mathbf{C}^{2}$ sur un autre, noté $U_{2}$.

2) $f_{2}(w, 0) \equiv 0$ et $f_{1}(0,0)=0$.

Alors l'une des trois possibilités suivantes est vérifiée:

i) $f_{1}(w, z)=\Gamma w ;(\Gamma>0)$ et $f_{2}(w, z)=f_{2}(z)$.

ii) $f_{1}(w, z)=\Gamma w ;(\Gamma>0)$ et $P_{2}(z, \bar{z})=P_{2}(|z|,|z|)$.

iii) $f_{1}(w, z)=\frac{\Gamma w}{1+i \lambda w} ;\left(\Gamma>0\right.$ et $\left.\lambda \in \mathbf{R}^{*}\right)$ et $P_{2}(z, \bar{z})=M|z|^{2 m}+Q$ avec $M>0, m \in \mathbf{N}^{*}$ et $Q \equiv 0$ ou $Q$ ne contient que des termes de degré $>2 m$.

On améliorera le contenu de cette proposition en établisant le Théorème 2 (cf. Section 4).

Dans cette partie, nous adoptons les notations suivantes:

Soit $P(z, \bar{z}) \in \mathcal{P}, P(z, \bar{z})$ peut s'écrire sous la forme $P(z, \bar{z})=$ $\sum_{l=m}^{N}|z|^{2 l} \operatorname{Re} V_{l}(z)$ où $V_{l}(z)=\sum_{j=0}^{N_{l}} \alpha_{j l} z^{j}$. Notons alors

$$
\begin{aligned}
V(z) & =: V_{m}(z) . \\
V^{*}(z) & =: V(z)-V(0) . \\
W(z) & =: z \frac{\partial V}{\partial z}(z) .
\end{aligned}
$$

Lorsque la partie homogène de plus bas degré de $P$ n'est pas équilibrée (et donc $\operatorname{Re} V(0)=0$ ) on dira que $P \in \tilde{\mathcal{P}}$, "est équilibrée" veut dire "est de la forme $M|z|^{2 m}, M \in \mathbf{R}^{*}, m \in \mathbf{N}^{*}$.

La proposition résultera des deux lemmes techniques suivants:

Lemme 3.2. Soient $\gamma \in \mathbf{R}, P(z, \bar{z}) \in \mathcal{P}$ et $A(z)$ une fonction holomorphe nulle à l'origine. 
Si les termes de la forme $|z|^{2 m} z^{p}$ ou $|z|^{2 m} \bar{z}^{q}$ où $(p, q \geq 0)$ sont identiquement nuls dans l'expression

$$
-\gamma P(z, \bar{z})+\operatorname{Re}\left(A(z) \cdot \frac{\partial P}{\partial z}(z, \bar{z})\right)
$$

on a alors:

$$
A(z)=z \frac{(2 \gamma-m \bar{a}) V^{*}(z)+2 m \cdot a \operatorname{Re} V(0)}{W(z)+m\left(V^{*}(z)+2 \operatorname{Re} V(0)\right)}, \text { avec } a=A^{\prime}(0) .
$$

Lemme 3.3. Soit $f: \Omega_{1} \rightarrow \Omega_{2}$ une application holomorphe propre satisfaisant les hypothèses de la Proposition 3.1 et soit $\vec{X}=f_{*}\left(i \frac{\partial}{\partial w}\right)$.

Supposons que sur un voisinage $U_{2}$ de l'origine le champ $\vec{X}$ soit donné par: $\vec{X}_{(w, z)}=: A(w) \frac{\partial}{\partial w}+B(z)(a w+b) \frac{\partial}{\partial z}$, où $(a, b) \in \mathbf{C}^{2}$, A étant une fonction entière et $B$ une fraction rationnelle. Alors $B$ est un polynôme holomorphe.

Ces deux lemmes seront démontrés ultérieurement.

Preuve de la Proposition 3.1: Començons par montrer que $f_{1}$ est de la forme générale $f_{1}=\frac{\Gamma w}{1+i \lambda w}$ où $\Gamma>0$ et $\lambda \in \mathbf{R}$. D'après la Proposition 2.1, on a $f_{1}(w, z)=f_{1}(w)$. Notons $D=:\{w \in \mathbf{C}: \operatorname{Re} w<0\}$; $f$ étant un biholomorphisme local au voisinage de l'origine, d'après la Proposition 1.1 , on a $J_{f}(w, 0) \neq 0$ pour tout $w \in D$. Donc $f_{1}$ est un biholomorphisme local sur $D$. D'autre part, $f_{1}$ est une application holomorphe propre de $D$ sur lui-même et donc $f_{1}$ est un revêtement fini de $D$ sur lui-même. Comme $D$ est simplement connexe, $f_{1}$ est donc un automorphisme de $D$. Il s'ensuit que $f_{1}(w)=\frac{\Gamma w}{1+i \lambda w}$ où $\Gamma>0$ et $\lambda \in \mathbf{R}$ puisque $f_{1}(0)=0$.

Passons maintenant à la preuve de i) et ii). D'après ce qui précède, si $\lambda=0$, on a $f_{1}(w)=\Gamma w,(\Gamma>0)$. Notons dans ce cas $f=\left(\Gamma w, f_{2}(w, z)\right)$ et considérons le champ $f_{*}\left(i \frac{\partial}{\partial w}\right)$. C'est un champ de vecteurs holomorphe défini sur $U_{2}$ et tangent à $b \Omega_{2}$. D'après la Proposition 2.1, $f_{*}\left(i \frac{\partial}{\partial w}\right)$ est de la forme $\left[f_{*}\left(i \frac{\partial}{\partial w}\right)\right]_{(w, z)}=i \Gamma \frac{\partial}{\partial w}+B(z) \frac{\partial}{\partial z}$, où $B$ est une fonction holomorphe dans un voisinage de l'origine, et on a $B(z)=i \beta z+\cdots+(\beta \in \mathbf{R})$ avec $\beta \neq 0$ si et seulement si $B \not \equiv 0$.

D'autre part, on a $\left[f_{*}\left(i \frac{\partial}{\partial w}\right)\right]_{f(w, z)}=i \Gamma \frac{\partial}{\partial w}+i \frac{\partial f_{2}}{\partial w}(w, z) \frac{\partial}{\partial z}$, d'où $(B \circ$ $\left.f_{2}\right)(w, z)=i \frac{\partial f_{2}}{\partial w}(w, z)$.

Si $B \equiv 0$, alors $\frac{\partial f_{2}}{\partial w}(w, z) \equiv 0$ et $f_{2}(w, z)=f_{2}(z)$ ce qui correspond au cas i). Supposons maintenant $B \not \equiv 0$. Puisque $i \Gamma \frac{\partial}{\partial w}$ est tangent à $b \Omega_{2}$, $B(z) \frac{\partial}{\partial z}$ l'est également. 
Cela se traduit par: $\operatorname{Re}\left(B(z) \frac{\partial}{\partial z}\left(\operatorname{Re} w+P_{2}(z, \bar{z})\right)\right)=0$ pour tout $(w, z)$ tel que $\operatorname{Re} w+P_{2}(z, \bar{z})=0$ ou encore par:

$$
\operatorname{Re}\left[B(z) \cdot \frac{\partial P_{2}}{\partial z}(z, \bar{z})\right]=0
$$

pour $z$ assez voisin de l'origine.

D'après le Lemme 3.2 , on a alors,

$$
B(z)=i \beta m z \frac{V^{*}(z)+2 \operatorname{Re} V(0)}{W(z)+m\left(V^{*}(z)+2 \operatorname{Re} V(0)\right)} .
$$

Or, d'après le Lemme 3.3, $B$ est un polynôme et, comme $d^{0} V^{*}=d^{0} W$, on a $B(z)=i \beta z, \beta \in \mathbf{R}^{*}$.

L'équation (1) devient $\operatorname{Re}\left[i \beta z \cdot \frac{\partial P_{2}}{\partial z}(z, \bar{z})\right]=0$, ce qui entraîne $P_{2}(z, \bar{z})=P_{2}(|z|,|z|)$ puisque

$$
\operatorname{Re}\left[i \beta z \frac{\partial}{\partial z} \sum_{p, q \geq 1} A_{p q} z^{p} \bar{z}^{q}\right]=\frac{i \beta}{2} \sum_{p, q \geq 1} A_{p q}(p-q) z^{p} \bar{z}^{q} .
$$

Pour la preuve de iii), nous allons montrer que si $\lambda \neq 0$, alors $P_{2}(z, \bar{z})$ n'appartient pas à $\tilde{\mathcal{P}}$. Nous procédons par l'absurde et supposons que $P_{2}(z, \bar{z}) \in \tilde{\mathcal{P}}$. Rappelons qu'au voisinage de l'origine, on a

$$
\begin{aligned}
f(w, z) & =\left(f_{1}(w), f_{2}(w, z)\right. \\
f^{-1}(w, z) & =\left(F_{1}(w), F_{2}(w, z)\right)
\end{aligned}
$$

avec $f_{1}(w)=\frac{\Gamma w}{1+i \lambda w}$ et $F_{1}(w)=\frac{w}{\Gamma-i \lambda w}$.

Considérons le champ de vecteurs holomorphe

$$
\vec{X}=f_{*}\left(i \frac{\partial}{\partial w}\right)=: A(w, z) \frac{\partial}{\partial w}+B(w, z) \frac{\partial}{\partial z} .
$$

On a

$$
\vec{X}_{f(w, z)}=i \frac{\partial f_{1}}{\partial w} \frac{\partial}{\partial w}+i \frac{\partial f_{2}}{\partial w} \frac{\partial}{\partial z}
$$

d'où $A(w, z)=i\left(\frac{\partial f_{1}}{\partial w} \circ F\right)(w)=\frac{i}{\Gamma}(\Gamma-i \lambda w)^{2}$. Les champs $\vec{X}$ et $i \frac{\partial}{\partial w}$ sont holomorphes tangents (dans le sens évoqué juste avant la Proposition 2.2) à $b \Omega_{2}$ donc les crochets de Lie $\left[\vec{X}, i \frac{\partial}{\partial w}\right]$ et $\left[\left[\vec{X}, i \frac{\partial}{\partial w}\right], i \frac{\partial}{\partial w}\right]$ le sont également. 
Or, on a

$$
\left[\vec{X}, i \frac{\partial}{\partial w}\right]=2 \frac{\lambda i}{\Gamma}(\Gamma-\lambda i w) \frac{\partial}{\partial w}+i \frac{\partial B}{\partial w} \frac{\partial}{\partial z}
$$

et

$$
\left[\left[\vec{X}, i \frac{\partial}{\partial w}\right], i \frac{\partial}{\partial w}\right]=2 \frac{\lambda^{2} i}{\Gamma} \frac{\partial}{\partial w}-\frac{\partial^{2} B}{\partial w^{2}} \frac{\partial}{\partial z} .
$$

Puisque $2 i \frac{\lambda^{2}}{\Gamma} \frac{\partial}{\partial w}$ est tangent à $b \Omega_{2}, \frac{\partial^{2} B}{\partial w^{2}} \frac{\partial}{\partial z}$ l'est également. Comme par hypothèses, $P_{2} \in \tilde{\mathcal{P}}$, la Proposition 2.2 montre alors que $\frac{\partial^{2} B}{\partial w^{2}} \equiv 0$. On a ainsi:

$B(w, z)=A_{1}(z) \cdot w+A_{0}(z)$ où $A_{0}$ et $A_{1}$ sont deux fonctions holomorphes au voisinage de l'origine.

Le champ $\vec{X}$ étant holomorphe tangent à $b \Omega_{2}$, on a:

$\operatorname{Re}\left(\vec{X}\left(\operatorname{Re} w+P_{2}(z, \bar{z})\right)\right) \equiv 0$ pour tout $(w, z)$ tel que $\operatorname{Re} w+P_{2}(z, \bar{z})=0$ c'est-à-dire $\operatorname{Re}\left[\frac{i}{2 \Gamma}(\Gamma-i \lambda w)^{2}+\left(A_{1}(z) \cdot w+A_{0}(z)\right) \frac{\partial P_{2}}{\partial z}(z, \bar{z})\right] \equiv 0$ pour $w=-P_{2}(z, \bar{z})+i v,(z, v)$ variant dans un voisinage de $(0,0)$ dans $\mathbf{C} \times \mathbf{R}$. Cela se traduit par:

(1) $v\left(-\frac{\lambda^{2}}{\Gamma} P_{2}(z, \bar{z})+\operatorname{Re}\left[i A_{1}(z) \cdot \frac{\partial P_{2}}{\partial z}(z, \bar{z})\right]\right)$

$$
-\lambda P_{2}(z, \bar{z})+\operatorname{Re}\left[\left(A_{0}(z)-A_{1}(z) P_{2}(z, \bar{z})\right) \frac{\partial P_{2}}{\partial z}(z, \bar{z})\right] \equiv 0
$$

et l'on en déduit:

$$
-\frac{\lambda^{2}}{\Gamma} P_{2}(z, \bar{z})+\operatorname{Re}\left[i A_{1}(z) \cdot \frac{\partial P_{2}}{\partial z}(z, \bar{z})\right] \equiv 0
$$

et

$$
-\lambda P_{2}(z, \bar{z})+\operatorname{Re}\left[\left(A_{0}(z)-A_{1}(z) P_{2}(z, \bar{z})\right) \frac{\partial P_{2}}{\partial z}(z, \bar{z})\right] \equiv 0 .
$$

Désignons par $H_{2 m}(z, \bar{z})$ la partie homogène de plus bas degré dans $P_{2}(z, \bar{z})$. De $(2)$, on tire: $\operatorname{Re}\left[i A_{1}(0) \frac{\partial H_{2 m}}{\partial z}(z, \bar{z})\right] \equiv 0$. Ceci, d'après le Lemme 2.3 , entraîne $A_{1}(0)=0$. On peut alors appliquer le Lemme 3.2 à l'équation (2) et en déduire que:

$$
A_{1}(z)=-i\left(\frac{2 \lambda^{2}}{\Gamma}+i m \bar{a}_{1}\right) z \cdot \frac{V^{*}(z)}{W(z)+m V^{*}(z)} \text { où } a_{1}=A_{1}^{\prime}(0)
$$

(on a utilisé le fait que $\operatorname{Re} V(0)=0$ puisque $P_{2} \in \tilde{\mathcal{P}}$ ). 
D'autre part, $B\left(f_{1}(w), f_{2}(w, z)\right)=i \frac{\partial f_{2}}{\partial w}(w, z)$ donc $B(0,0)=0$ puisque $f_{2}(w, 0) \equiv 0$ et $f_{1}(0)=0$. On a donc $A_{0}(0)=0$. En observant que les termes de la forme $|z|^{2 m} z^{p}$ ou $|z|^{2 m} \bar{z}^{q}$ du membre gauche de (3) ne peuvent provenir que de $-\lambda P_{2}(z, \bar{z})+\operatorname{Re}\left[A_{0}(z) \frac{\partial P_{2}}{\partial z}(z, \bar{z})\right]$, on peut à nouveau utiliser le Lemme 3.2 et obtenir

$$
A_{0}(z)=\left(2 \lambda-m \bar{a}_{0}\right) z \frac{V^{*}(z)}{W(z)+m V^{*}(z)} \text { où } a_{0}=A_{0}^{\prime}(0) .
$$

En définitive $f_{*}\left(i \frac{\partial}{\partial w}\right)$ est donné par:

(6) $\quad f_{*}\left(i \frac{\partial}{\partial w}\right)=\frac{i}{\Gamma}(\Gamma-\lambda i w)^{2} \frac{\partial}{\partial w}+(a w+b) z \frac{V^{*}(z)}{W(z)+m V^{*}(z)} \frac{\partial}{\partial z}$

où $a$ et $b$ sont des constantes complexes. Nous pouvons donc appliquer le Lemme 3.3 et en déduire que $z \frac{V^{*}(z)}{W(z)+m V^{*}(z)}$ est un polynôme holomorphe. Comme $V^{*}$ et $W$ ont même degré, ce polynôme est de la forme $\alpha z, \alpha \in \mathbf{C}$. De (4) et (5), on tire alors:

$$
A_{0}(z)=a_{0} z \text { et } A_{1}(z)=a_{1} z
$$

Pour terminer, nous revenons à l'identité (2). Tenant compte de (7), on a:

$$
-\frac{\lambda^{2}}{\Gamma} P_{2}(z, \bar{z})+\operatorname{Re}\left[i a_{1} z \frac{\partial P_{2}}{\partial z}(z, \bar{z})\right] \equiv 0 .
$$

Posons $P_{2}(z, \bar{z})=\sum_{p, q \geq 1} A_{p q} z^{p} \bar{z}^{q}$; en reportant dans (8), on obtient:

$$
\sum_{p, q \geq 1} A_{p q}\left[-2 \frac{\lambda^{2}}{\Gamma}-(p+q) \operatorname{Im} a_{1}+i(p-q) \operatorname{Re} a_{1}\right] z^{p} \bar{z}^{q} \equiv 0 .
$$

Comme $\lambda \neq 0$ par hypothèse, on obtient facilement de (9) que $P_{2}$ est homogène c'est-à-dire $P_{2}=H_{2 m}$. Tenant compte de (7), l'identité (3) devient alors

$$
-\lambda H_{2 m}(z, \bar{z})+\operatorname{Re}\left[z\left(a_{0}-a_{1} H_{2 m}(z, \bar{z})\right) \frac{\partial H_{2 m}}{\partial z}(z, \bar{z})\right] \equiv 0 .
$$

On en déduit que:

$$
-\lambda H_{2 m}(z, \bar{z})+\operatorname{Re}\left(a_{0} z \frac{\partial H_{2 m}}{\partial z}(z, \bar{z})\right) \equiv 0
$$


et

$$
H_{2 m}(z, \bar{z}) \operatorname{Re}\left(a_{1} z \frac{\partial H_{2 m}}{\partial z}(z, \bar{z})\right) \equiv 0 \text {. }
$$

D'après le Lemme 2.3, (12) force $H_{2 m}$ a être équilibré. Ce qui est absurde.

Preuve du Lemme 3.2: On pose

$$
A(z)=z(a+S(z)) \text { où } S(z)=\sum_{k=2}^{+\infty} a_{k} z^{k-1} .
$$

En tenant compte des notations adoptées, les termes de la forme $|z|^{2 m} z^{p}$ ou $|z|^{2 m} \bar{z}^{q}(p, q \geq 0)$ dans (*) ne peuvent provenir que de:

$$
-\gamma\left(|z|^{2 m} \operatorname{Re} V(z)\right)+\operatorname{Re}\left[A(z) \cdot \frac{\partial}{\partial z}\left(|z|^{2 m} \operatorname{Re} V(z)\right)\right] .
$$

Par ailleurs, on a:

$$
A(z) \cdot \frac{\partial}{\partial z}\left(|z|^{2 m} \operatorname{Re} V(z)\right)=|z|^{2 m}(a+S)\left(m \operatorname{Re} V+\frac{1}{2} W\right)
$$

et

$$
\begin{aligned}
& \operatorname{Re}\left[(a+S)\left(m \operatorname{Re} V+\frac{1}{2} W\right)\right] \\
&=\left(\operatorname{Re} a+\frac{S+\bar{S}}{2}\right) \times\left(m\left(\frac{V^{*}+\bar{V}^{*}}{2}+\operatorname{Re} V(0)\right)+\frac{1}{4}(W+\bar{W})\right) \\
&+\left(i \operatorname{Im} a+\frac{S-\bar{S}}{2}\right)\left(\frac{W-\bar{W}}{4}\right) .
\end{aligned}
$$

Les termes harmoniques de (15) sont:

$$
\begin{aligned}
2 \operatorname{Re}\left[\frac { S } { 4 } \left(m V^{*}+\right.\right. & 2 m \operatorname{Re} V(0)+W)+\frac{a}{4} W \\
+ & \left.\frac{m}{2}(\operatorname{Re} a) \cdot V^{*}+m \frac{(\operatorname{Re} a) \operatorname{Re} V(0)}{2}\right]=: 2 \operatorname{Re}[K(z)] .
\end{aligned}
$$

Donc les termes de la forme $|z|^{2 m} z^{p}$ ou $|z|^{2 m} \bar{z}^{q}(p, q \geq 0)$ dans (13) sont: $|z|^{2 m} \operatorname{Re}[-\gamma V(z)+2 K(z)]$. Par hypothèse, ils sont identiquement nuls, d'où:

$$
-\gamma V(z)+2 K(z)=-\gamma V(0)+2 K(0)
$$


En remplaçant $K$ par son expression et $V$ par $V^{*}+V(0)$ dans (16), on obtient:

$$
\frac{S}{2}\left(m V^{*}+2 m \operatorname{Re} V(0)+W\right)+\frac{a}{2} W+V^{*}(m \operatorname{Re} a-\gamma)=0
$$

ou encore:

$$
S(z)=-\frac{2(m \operatorname{Re} a-\gamma) V^{*}(z)+a W(z)}{W(z)+m V^{*}(z)+2 m \operatorname{Re} V(0)}
$$

Puisque $A(z)=z(a+S(z))$, on a donc:

$$
A(z)=z \frac{(2 \gamma-m \bar{a}) V^{*}(z)+2 m a \operatorname{Re} V(0)}{W(z)+m V^{*}(z)+2 m \operatorname{Re} V(0)} \text { où } a=A^{\prime}(0) .
$$

Preuve du Lemme 3.3: Supposons que $B$ ait des pôles.

Soit $\mathcal{S}$ l'ensemble des pôles de $B$ dans $\Omega_{2}$, c'est-à-dire $\mathcal{S}=\Omega_{2} \cap$ $\left(B^{-1}(\infty)\right)$.

Soient $z_{1}$ un élément de $\mathcal{S}, w_{1}$ un nombre complexe tel que $\left(w_{1}, z_{1}\right) \in \mathcal{S}$ et, en outre, $a w_{1}+b \neq 0$. (Ce dernier choix est toujours possible quitte à faire une translation en $\operatorname{Im} w_{1}$.)

Soit $\left(w_{0}, z_{0}\right)$ un point fixé dans $U_{2} \backslash f\left(V_{f}\right)$; puisque $\Omega \backslash\left(f\left(V_{f}\right) \cup \mathcal{S}\right)$ est connexe et dense dans $\Omega_{2}$, il existe un chemin continu $\gamma:[0,1] \rightarrow \Omega_{2}$ tel que: $\gamma(0)=\left(w_{0}, z_{0}\right) ; \gamma(1)=\left(w_{1}, z_{1}\right)$ et $\forall t \in\left[0,1\left[, \gamma(t) \notin f\left(V_{f}\right) \cup \mathcal{S}\right.\right.$.

L'application $f$ étant holomorphe propre, $f: \Omega_{1} \backslash f^{-1}\left[f\left(V_{f}\right)\right] \rightarrow$ $\Omega_{2} \backslash f\left(V_{f}\right)$ est un revêtement fini, il existe donc $\tilde{\gamma}:\left[0,1\left[\rightarrow \Omega_{1} \backslash V_{f}\right.\right.$ un relèvement de $\gamma$ par $f$ c'est-à-dire $\forall t \in[0,1[,(f \circ \tilde{\gamma})(t)=\gamma(t)$.

Par hypothèse, au voisinage de l'origine le champ de vecteurs holomorphes $\vec{X}=f_{*}\left(i \frac{\partial}{\partial w}\right)$ est de la forme: $\vec{X}_{(w, z)}=A(w) \frac{\partial}{\partial w}+B(z)(a w+b) \frac{\partial}{\partial z}$ où $A$ est une fonction entière et $B$ est une fraction rationnelle.

L'existence du relèvement $\tilde{\gamma}$ permet de prolonger holomorphiquement $f_{*}\left(i \frac{\partial}{\partial w}\right)$ le long de $\gamma([0,1[)$ en un champ $\overrightarrow{\tilde{X}}$ tel que

$$
\forall t \in\left[0,1\left[: \overrightarrow{\tilde{X}}_{\gamma(t)}=\left[f_{*}\left(i \frac{\partial}{\partial w}\right)\right]_{\gamma(t)}=i \frac{\partial f_{1}}{\partial w}(\tilde{\gamma}(t)) \frac{\partial}{\partial w}+i \frac{\partial f_{2}}{\partial w}(\tilde{\gamma}(t)) \frac{\partial}{\partial z}\right. \text {. }\right.
$$

Par ailleurs, puisque $\gamma\left(\left[0,1[) \subset \Omega_{2} \backslash \mathcal{S}\right.\right.$, le champ $A(w) \frac{\partial}{\partial w}+B(z)(a w+$ b) $\frac{\partial}{\partial z}$ est également un prolongement de $\vec{X}$ le long de $\gamma([0,1[)$.

Par unicité du prolongement, on a donc:

$$
\forall t \in\left[0,1\left[, i \frac{\partial f_{1}}{\partial w}(\tilde{\gamma}(t))=A\left(\gamma_{1}(t)\right)\right.\right.
$$

$$
\forall t \in\left[0,1\left[: i \frac{\partial f_{2}}{\partial w}(\tilde{\gamma}(t))=B\left(\gamma_{2}(t)\right)\left(a \gamma_{1}(t)+b\right) \text { où } \gamma=\left(\gamma_{1}, \gamma_{2}\right) .\right.\right.
$$

Comme $\left(a w_{1}+b\right) \neq 0$, on déduit immédiatement de (19) que $\left|B\left(z_{1}\right)\right|<$ $+\infty$ ce qui est impossible. 


\section{Preuve des Théorèmes 1 et 2}

Preuve du Théorème 2: On se place dans le cas iii) de la Proposition 3.1. La partie homogène de plus bas degré de $P_{2}$ est égale à $M|z|^{2 m}$ $(M>0)$ et comme le montre un calcul élémentaire celle de $P_{1}$ est aussi égale à $M^{\prime}|z|^{2 m}\left(M^{\prime}>0\right)$. Notons $2 k$ le degré du polynôme $P_{2}$ et $H_{2 k}$ sa partie homogène de plus haut degré, il nous faut montrer que $k=m$.

Nous utiliserons pour cela la méthode de dilatation des coordonnées. Considérons à cet effet la suite de points de $\Omega_{1}:\left(-\frac{1}{n}+\frac{i}{\lambda}, 0\right)$, la suite de points de $\Omega_{2}: f\left(-\frac{1}{n}+\frac{i}{\lambda}, 0\right)=\left(-\frac{i \Gamma}{\lambda}-\frac{n \Gamma}{\lambda^{2}}, 0\right)$ et deux suites de dilatations $\left(S_{n}\right)_{n}$, et $\left(\Delta_{n}\right)$ définies par:

$$
\begin{aligned}
S_{n}(w, z) & =:\left(n w, z n^{\frac{1}{2 m}}\right) . \\
\Delta_{n}(w, z) & =: \frac{\lambda^{2}}{\Gamma}\left(\frac{w}{n}, \frac{z}{n^{\frac{1}{2 k}}}\right) .
\end{aligned}
$$

Soit $h$ l'application holomorphe propre de $\Omega_{1}$ sur $\Omega_{2}$ donnée par:

$$
\begin{aligned}
h: \Omega_{1} & \rightarrow \Omega_{2} \\
(w, z) & \mapsto f\left(w+\frac{i}{\lambda}, z\right)+\left(\frac{\Gamma i}{\lambda}, 0\right) .
\end{aligned}
$$

On définit alors une suite d'applications holomorphes propres $\left(F_{n}\right)_{n}$ de $S_{n}\left(\Omega_{1}\right)$ sur $\Delta_{n}\left(\Omega_{2}\right)$ par $F_{n}=\Delta_{n} \circ h \circ S_{n}^{-1}$.

On vérifie sans peine que:

1) $F_{n}(w, z)=\left(\frac{1}{w}, \frac{\lambda^{2}}{\Gamma} n^{-\frac{1}{2 k}} f_{2}\left(\frac{w}{n}+\frac{i}{\lambda}, z n^{-\frac{1}{2 m}}\right)\right)$.

2) Pour tout $n: F_{n}(w, 0)=\left(\frac{1}{w}, 0\right)$ et $F_{n}(-1,0)=(-1,0)$.

3) Pour tout $n$, la multiplicité de $F_{n}$ est égale à celle de $f$.

4) $S_{n}\left(\Omega_{1}\right)$ converge vers $D_{1}=:\left\{(w, z) \in \mathbf{C}^{2}: \operatorname{Re} w+M^{\prime}|z|^{2 m}<0\right\}$.

5) $\Delta_{n}\left(\Omega_{2}\right)$ converge vers $D_{2}=:\left\{(w, z) \in \mathbf{C}^{2}: \operatorname{Re} w+\left(\frac{\Gamma}{\lambda}\right)^{2 k-1} H_{2 k}(z, \bar{z})<\right.$ $0\}$.

D'autre part, d'après ([7, Lemme 2.3$])$ et après une éventuelle extraction, la suite $\left(F_{n}\right)_{n}$ converge uniformémemnt sur tout compact de $D_{1}$ vers une application holomorphe: $F: D_{1} \rightarrow D_{2}$ telle que:

a) $F(w, z)=\left(\frac{1}{w}, F_{2}(w, z)\right)$.

b) $F_{2}(w, z) \equiv 0$.

Admettons momentanément que pour $w$, fixé, $F_{2}(w, \cdot)$ est surjective finie. Il en va alors de même pour $F$. Montrons que $F$ est propre. Posons:

$$
\begin{aligned}
& \rho_{1}(w, z)=: \operatorname{Re} w+M^{\prime}|z|^{2 m} \\
& \rho_{2}(w, z)=: \operatorname{Re} w+\left(\frac{\Gamma}{\lambda}\right)^{2 k-1} H_{2 k}(z, \bar{z}) .
\end{aligned}
$$


On a:

$$
\begin{aligned}
& D_{1}=\left\{(w, z) \in \mathbf{C}^{2}: \rho_{1}(w, z)<0\right\} \\
& D_{2}=\left\{(w, z) \in \mathbf{C}^{2}: \rho_{1}(w, z)<0\right\}
\end{aligned}
$$

sur $D_{2}$, on définit la fonction $\sigma(w, z)=: \sup _{F(u, v)=(w, z)} \rho_{1}(u, v)$. Puisque $F$ est surjective et finie $\sigma$ est p.s.h. et strictement négative sur $D_{2}$.

Supposons qu'il existe une suite $\left(w_{n}, z_{n}\right)_{n}$ de points de $D_{1}$ qui converge vers un point de $b D_{1}$ et telle que $\left(F\left(w_{n}, z_{n}\right)\right)_{n}$ converge vers un point de $D_{2}$. Deux cas sont à distinguer. Commençons par supposer que la limite de $\left(w_{n}, z_{n}\right)$ est finie, soit $\left(w_{0}, z_{0}\right)$ cette limite et soit $\left(w_{1}, z_{1}\right)$ la limite de $\left(F\left(w_{n}, z_{n}\right)\right)_{n}$. On a: $\sigma\left(F\left(w_{n}, z_{n}\right)\right) \geq \rho_{1}\left(w_{n}, z_{n}\right)$, et puisque $\sigma$ est (s.c.s.) $\sigma\left(w_{1}, z_{1}\right) \geq \rho_{1}\left(w_{0}, z_{0}\right)=0$. Ce qui est absurde puisque $\sigma$ est strictement négative. Supposons maintenant que $\left(\left|w_{n}\right|+\left|z_{n}\right|\right) \rightarrow+\infty,\left(w_{n}, z_{n}\right)_{n}$ étant dans $D_{1}$ on a $\left|w_{n}\right| \rightarrow+\infty$ et d'après a) $F\left(w_{n}, z_{n}\right) \rightarrow(0, z)$, par hypothèse $(0, z) \in D_{2}$ donc il existe $N \in \mathbf{N}^{*}$ tel que pour $n \geq N:(0, z) \in \Delta_{n}\left(\Omega_{2}\right)$. Comme $F_{n}$ est propre, il existe $(u, v) \in S_{n}\left(\Omega_{1}\right)$ tel que $F_{n}(u, v)=(0, z)$, d'après 1 ) ceci est impossible. Nous avons donc montré que $F$ est propre. Etablissons maintenant que $m \geq k$.

Soit $\left(w_{n}, 0\right)$ une suite de points de $D_{1}$ qui converge vers un point $\left(w_{0}, 0\right)$ de $b D_{1}$ où $w_{0} \neq 0$. La suite $\left(F\left(w_{n}, 0\right)\right)_{n}$ converge vers le point $\left(\frac{1}{w_{0}}, 0\right)$ de $b D_{2}$, le bord de $D_{2}$ étant de type fini, on sait d'après $[6]$ que l'application $F$ se prolonge continûment à $\bar{D}_{1}$ sur un voisinage de $\left(w_{0}, 0\right)$, et d'après Bell et Catlin $[\mathbf{5}] F$ se prolonge différentiablement à $\bar{D}_{1}$ sur un voisinage de $\left(w_{0}, 0\right)$. Donc si $\tau_{1}$ (respectivement $\tau_{2}$ ) désigne la fonction type de $b D_{1}$ (respectivement de $b D_{2}$ ) alors:

$$
\tau_{1}\left(w_{0}, 0\right) \geq \tau_{2}\left(F\left(w_{0}, 0\right)\right)=\tau_{2}\left(\frac{1}{w_{0}}, 0\right)
$$

d'où $m \geq k$.

Ceci termine la preuve du Théorème 2 .

Montrons maintenant que pour $w$ fixé, $F_{2}(w, \cdot)$ est finie.

On vérifie facilement que:

$$
S_{n}\left(\Omega_{1}\right)=:\left\{(w, z) \in \mathbf{C}^{2}: \operatorname{Re} w+M^{\prime}|z|^{2 m}+Q_{n}(z, \bar{z})<0\right\}
$$

et

$$
\Delta_{n}\left(\Omega_{2}\right)=:\left\{(w, z) \in \mathbf{C}^{2}: \operatorname{Re} w+\left(\frac{\Gamma}{\lambda}\right)^{2 k-1} H_{2 k}(z, \bar{z})+\tilde{Q}_{n}(z, \bar{z})<0\right\}
$$


où $Q_{n}(z, \bar{z})$ et $\tilde{Q}_{n}(z, \bar{z})$ sont des polynômes qui convergent uniformément sur tout compact de $\mathbf{C}$ vers le polynôme nul.

Soit $w \in \mathbf{C}^{*}$, un point fixé tel que $\operatorname{Re} w<0$. On notera:

$$
\begin{aligned}
\Omega_{n, w} & =:\left\{z \in \mathbf{C}: M^{\prime}|z|^{2 m}+Q_{n}(z, \bar{z})<-\operatorname{Re} w\right\} . \\
D_{n, w} & =:\left\{z \in \mathbf{C}:\left(\frac{\Gamma}{\lambda}\right)^{2 k-1} H_{2 k}(z, \bar{z})+\tilde{Q}_{n}(z, \bar{z})<-\frac{\operatorname{Re} w}{|w|^{2}}\right\} . \\
\Omega_{\infty, w} & =:\left\{z \in \mathbf{C}: M^{\prime}|z|^{2 m}<-\operatorname{Re} w\right\} . \\
D_{\infty, w} & =:\left\{z \in \mathbf{C}:\left(\frac{\Gamma}{\lambda}\right)^{2 k-1} H_{2 k}(z, \bar{z})<-\frac{\operatorname{Re} w}{|w|^{2}}\right\} . \\
\Delta(0, R) & =:\{z \in \mathbf{C}:|z|<R\} .
\end{aligned}
$$

$F_{n}=:\left(\frac{1}{w} ; F_{n, 2}\right)$ la suite d'applications holomorphes propre de $S_{n}\left(\Omega_{1}\right)$ sur $\Delta_{n}\left(\Omega_{2}\right)$.

Soit $R>0$ tel que $\operatorname{Re} w+R^{2 m}>0$. Pour $n$ assez grand, on a $\Omega_{n, w} \cap\{|z|=R\}=\emptyset$, on en déduit que la composante connexe de l'origine de $\Omega_{n, w}$ est contenue dans $\{|z|<R\}$. Notons $\Omega_{n, w}^{0}$ cette composante, il est clair que $\Omega_{n, w}^{0}$ converge vers $\Omega_{\infty, w}$. La composante connexe, $D_{n, w}^{0}$, de l'origine dans $D_{n, w}$ conïncide avec $F_{n}\left(\Omega_{n, w}^{0}\right)$. En observant que $D_{\infty, w}$ est connexe (étoilé par rapport à l'origine) on vérifie facilement que $D_{n, w}^{0}$ converge vers $D_{\infty, w}$.

Ainsi la suite d'applications holomorphes propres

$$
\begin{aligned}
h_{n}: \Omega_{n, w}^{0} & \rightarrow D_{n, w}^{0} \\
z & \mapsto F_{n, 2}(w, z)
\end{aligned}
$$

est telle que (modulo extraction)

1) $h_{n}(0)=0, \forall n$.

2) $h_{n}$ est de multiplicité finie.

3) $h_{n}$ converge vers $F_{2}(w, \cdot): \Omega_{\infty, w} \rightarrow D_{\infty, w}$ uniformément sur tout compact, et il existe $R>0$ tel que $\Omega_{n, w}^{0} \subset\{|z|<R\}$ pour tout $n$.

On en déduit par des arguments standards que $F_{2}(w, \cdot)$ est surjective et finie.

Preuve du Théorème 1: Nous supposons $V_{f} \neq \emptyset$ et montrons que cela conduit à une contradiction, nous procédons en trois étapes.

1ère étape: Il existe deux automorphismes de $\mathbf{C}^{2}, \varphi_{1}$ et $\varphi_{2}$ tels que l'application $\tilde{f}=\varphi_{1}^{-1} \circ f \circ \varphi_{2}$ satisfait les hypothèses du Théorème 2 .

Pour tout $z \in \mathbf{C}$, notons $\pi_{z}$ le demi-plan $\{(w, z): w \in \mathbf{C}\} \cap \Omega$, et désignons par $f^{n}$ la $n^{\text {ième }}$ itérée de $f$. Comme $V_{f}$ est supposé non vide, 
la Proposition 1.1 assure l'existence d'un demi-plan $\pi_{z_{0}}$ contenu dans $V_{f}$. En utilisant l'inclusion $f^{-1}\left(V_{f^{n}}\right) \subset V_{f^{n+1}}$ et le fait que, toujours d'après la Proposition 1.1, $V_{f^{n}}$ est une réunion de demi-plan, on construit par récurrence une suite $\left(z_{n}\right)_{n}$ de nombres complexe telle que:

i) $\forall n \geq 1: \pi_{z_{n}} \subset V_{f^{n}}$.

ii) $\forall n \geq 1: f\left(\pi_{z_{n+1}}\right) \subset \pi_{z_{n}}$.

On observera pour cela que: si $\left(w_{n+1}, z_{n+1}\right) \in f^{-1}\left(\pi_{z_{n}}\right) \cap \pi_{z_{n+1}}$ alors $f\left(\pi_{z_{n+1}}\right) \subset \pi_{z_{n}}$. En effet, dans le cas contraire, $w_{n+1}$ serait un zéro isolé de $f_{2}\left(w, z_{n+1}\right)-z_{n}$ et $\left(w_{n+1}, z_{n+1}\right)$ serait un zéro isolé de $f_{2}(w, z)-z_{n}$ puisque $f^{-1}\left(\pi_{z_{n}}\right)$ est contenu dans une réunion de demi-plan $\pi_{z}$.

Notons $\tau_{n}$ la valeur de $\tau$ sur le bord de $\pi_{z_{n}}$. Il résulte de ii) et des propiétés de $\tau$ que la suite $\left(\tau_{n}\right)_{n \geq 1}$ est croissante. Comme les valeurs de $\tau$ sont entières et majorées par le degré de $P$. Il existe $n_{0} \in \mathbf{N}^{*}$ tel que $\tau_{n_{0}+1}=\tau_{n_{0}}$. Pour fixer les idées, nous supposons que $n_{0}=1$. Alors $f\left(\pi_{z_{2}}\right) \subset \pi_{z_{1}}$ et, puisque $\tau_{2}=\tau_{1}, \pi_{z_{2}} \not \subset V_{f}$. Quitte à composer $f$ avec des translations $(w, z) \mapsto\left(w+i t_{0}, z\right)$, on peut supposer que $f$ induit un difféomorphisme local sur $b \Omega$ au voisinage de $\left(-P\left(z_{2}\right), z_{2}\right)$ et que $f\left(-P\left(z_{2}\right), z_{2}\right)=\left(-P\left(z_{1}\right), z_{1}\right)$. Définissons les automorphismes de $\mathbf{C}^{2}, \varphi_{1}$ et $\varphi_{2}$, par:

$$
\varphi_{j}(w, z)=\left(w^{\prime}, z^{\prime}\right)
$$

où

$$
\left\{\begin{array}{l}
w^{\prime}=w-P\left(z_{j}, \bar{z}_{j}\right)-2 \sum_{i=1}^{2 m} \frac{1}{i !} \frac{\partial^{i} P}{\partial z^{i}}\left(z_{j}, \bar{z}_{j}\right) z^{i} . \\
z^{\prime}=z+z_{j} .
\end{array}\right.
$$

Ils induisent des automorphismes de $\Omega_{j}$ sur $\Omega$ où

$$
\begin{aligned}
\Omega_{j} & =:\left\{(w, z) \in \mathbf{C}^{2}: \operatorname{Re} w+Q_{j}(z, \bar{z})<0\right\} \\
Q_{j}(z, \bar{z}) & =P\left(z+z_{j}, \bar{z}+\bar{z}_{j}\right)-P\left(z_{j}, \bar{z}_{j}\right)-2 \operatorname{Re} \sum_{i=1}^{2 m} \frac{\partial^{i} P}{\partial z^{i}}\left(z_{j}, \bar{z}_{j}\right) z^{i} .
\end{aligned}
$$

Par construction, l'application $\tilde{f}=\varphi_{1}^{-1} \circ f \circ \varphi_{2}$ est propre de $\Omega_{2}$ sur $\Omega_{1}$, fixe la droite complexe $z=0$ et l'origine $(0,0)$. D'après $[\mathbf{1 1}], \tilde{f}$ se prolonge en un biholomorphisme local au voisinage de $(0,0)$.

2ème étape: $V_{f}$ est contenu dans une réunion d'au plus $(2 m)=\operatorname{deg} P$ droites complexes.

Soit $\tilde{f}$ l'application fournie par la première étape. D'après le Théorème 2, deux cas sont à distinguer. Commençons par supposer que $\tilde{f}$ est de la forme $\left(\Gamma w, \tilde{f}_{2}(z)\right)$. Alors $\tilde{f}_{2}$ est une fonction entière telle 
que $\tilde{f}_{2}(0)=0$ et $\frac{\partial \tilde{f}_{2}}{\partial z}(0)=\lambda \neq 0$. L'inclusion $\tilde{f}\left(b \Omega_{2}\right) \subset b \Omega_{1}$ se traduit au voisinage de l'origine par l'identité:

$$
\Gamma Q_{2}(z, \bar{z})=Q_{1} \circ \tilde{f}_{2}(z)
$$

Décomposons les polynômes $Q_{1}$ et $Q_{2}$ sous la forme suivante

$$
Q_{j}(z, \bar{z})=\bar{z}^{k_{j}}\left[h_{j}(z)+\bar{z} R_{j}(z, \bar{z})\right]
$$

où $k_{j} \in \mathbf{N}^{*}, h_{j}$ est un polynôme holomorphe et $R_{j}$ un polynôme en $z, \bar{z}$.

En identifiant les termes de plus bas degré en $\bar{z}$ dans (20), on obtient:

$$
\Gamma \bar{z}^{k_{2}} h_{2}(z)=\bar{\lambda}^{k_{1}} \bar{z}^{k_{1}} h_{1}\left(\tilde{f}_{2}(z)\right)
$$

On déduit alors de (21) que $k_{1}=k_{2}=: k$ et

$$
\Gamma h_{2}(z)=\bar{\lambda}^{k} h_{1} \circ \tilde{f}_{2}(z) .
$$

Ceci montre que le nombre de zéros de $\frac{\partial \tilde{f}_{2}}{\partial z}$ est majoré par le degré de $h_{2}$ et donc par $2 m$. Il s'ensuit que $V_{\tilde{f}}$ est constitué d'au plus $2 m$ demi-plans de la forme $\pi_{z}$. Il en va de même pour $V_{f}$, puisque les automorphismes $\varphi_{1}$ et $\varphi_{2}$ échangent ce type de demi-plans.

Il nous reste à envisager les cas où $Q_{1}$ ne dépend que de $|z|$. Considérons l'application holomorphe propre de $\Omega_{1}$ sur lui-même définie par $g=: \varphi_{1}^{-1} \circ f \circ \varphi_{1}$.

Si $\left(w_{0}, z_{0}\right) \in \bar{V}_{g} \cap b \Omega_{1}$, alors d'après la Proposition 1.1, on a $\pi_{z_{0}} \subset V_{g}$. Quitte à composer $g$ avec une translation $(w, z) \rightarrow(w+i y, z)$, on peut supposer que $g$ se prolonge différentiablement au voisinage de $\left(w_{0}, z_{0}\right)$. Alors pour $\varepsilon>0$ assez petit, on a:

$$
\left\{\left(w_{0}, e^{i t} z_{0}\right), t \in[-\varepsilon, \varepsilon]\right\} \subset \bar{V}_{g} \cap b \Omega_{1} \text {. }
$$

En effet, sinon, on trouverait une suite $\left(t_{n}\right)_{n \in N}$ telle que $\lim _{n} t_{n}=0$ et $J g\left(w_{0}, e^{i t_{n}}\right) \neq 0$.

Pour tout $n \geq 1$ : on aurait alors $\tau\left[g\left(w_{0}, e^{i t_{n}} z_{0}\right)\right]=\tau\left(w_{0}, e^{i t_{n}} z_{0}\right)=$ $\tau\left(w_{0}, z_{0}\right)$ et $\tau$ étant s.c.s., $\tau\left(w_{0}, z_{0}\right) \leq \tau\left(g\left(w_{0}, z_{0}\right)\right)$. Comme, par ailleurs, $\tau\left(g\left(w_{0}, z_{0}\right)\right) \leq \tau\left(w_{0}, z_{0}\right)$, il en résulterait que $\tau\left(g\left(w_{0}, z_{0}\right)\right)=\tau\left(w_{0}, z_{0}\right)$ et donc que $J g\left(w_{0}, z_{0}\right) \neq 0$, ce qui est contraire aux hypothèses.

Donc si $z_{0} \neq 0,(23)$ contredit la Proposition 1.1, donc $V_{g}=\pi_{0}$ ou encore $V_{f}=\pi_{z_{1}}$. 
3ème étape: Conclusion: D'après la 2ème étape, les lieux de branchement $V_{f^{n}}$ ont au plus $2 m$ composantes connexes. Comme $V_{f^{n+1}}=$ $V_{f^{n}} \cup f^{-1}\left(V_{f^{n}}\right) \supset V_{f^{n}}$, il s'ensuit que la suite $\left(V_{f^{n}}\right)$ est stationnaire.

On supposera sans perte de génétalité que $V_{f}=V_{f^{2}}$, ce qui signifie que $f^{-1}\left(V_{f}\right) \subset V_{f}$. Notons $\pi_{1}, \ldots, \pi_{N}$ les composantes connexes de $V_{f}$. Puisque $f^{-1}\left(\pi_{j}\right)$ est un ensemble analytique dans $\Omega$ contenu dans $V_{f}$ il existe $\sigma(j) \in\{1, \ldots, N\}$ tel que $\pi_{\sigma(j)} \subset f^{-1}\left(\pi_{j}\right)$, on voit alors facilement que $f$ iduit une permutation sur $\left\{\pi_{1}, \ldots, \pi_{N}\right\}$. Alors $f^{N !}\left(\pi_{1}\right) \subset \pi_{1}$ et donc, d'après la preuve du Lemme $1.2, \pi_{1} \not \subset V_{f^{N !}}$. Ainsi $V_{f}=\emptyset$ et, $\Omega$ étant simplement connexe, $f$ est un automorphisme.

\section{References}

1. H. Alexander, Holomorphic mappings from the ball and polydisc, Math. Ann. 209 (1974), 249-256.

2. E. Bedford And S. Bell, Proper self-maps of weakly pseudoconvex domains, Math. Ann. 261 (1982), 47-49.

3. E. Bedford and J. E. Fornaess, A construction of peak functions on weakly pseudoconvex domains, Ann. of Math. 107 (1978), $555-568$.

4. E. Bedford And S. Pinchuk, Domains in $\mathbb{C}^{2}$ with non compact holomorphic automorphism groups, Math. USSR Sbornik 63 (1989), 141-151.

5. S. Bell and D. Catlin, Regularity of $C R$ mappins, Math. Z. 199 (1988), 357-368.

6. F. Berteloot, A remark on local continuous extension of proper holomorphic mappings, Contemporary Mathematics 137 (1992), 79-83.

7. F. Berteloot, Characterization of models in $\mathbb{C}^{2}$ by their automorphism groups, Int. J. Mathematics 5(5) (1994), 619-634.

8. F. Berteloot and S. Pinchuk, Proper holomorphic mappings between bounded complete Reinhardt domains in $\mathbb{C}^{2}$, Math. Z. 219 (1995), 343-356.

9. A. Chaouech, Une remarque sur un résultat de Y. Pan, Ann. Fac. Sci. Toulouse 5 (1996), 1-4.

10. K. Diederich and J. E. Fornaess, Proper holomorphic images of strictly pseudoconvex domains, Math. Ann. 259 (1982), 279-286.

11. K. Diederich And J. Fornaess, Proper holomorphic mappings between real analytic pseudoconvex domains in $\mathbb{C}^{n}$, Math. Ann. 382 (1988), 681-700. 
12. Y. PAN, Proper holomorphic self-mappings of Reinhardt domains, Math. Z. 208 (1991), 289-295.

13. S. Pinchuk, On proper maps of strictly pseudoconvex domains, Siberian Math. J. 15 (1974), 909-917.

U.F.R. de Mathématiques

Université de Lille

URA C.N.R.S. D751

F-59655 Villeneuve d'Ascq Cedex

FRANCE

Rebut el 22 de Desembre de 1994 